\title{
Análisis de infraestructura, gestión de Colecciones y desempeño del personal de los museos de la provincia del Carchi
}

\section{Analysis of infrastructure, management of collections and performance of staff of the Museums of the Province of Carchi}

\author{
Yajaira Lisseth Chávez Sánchez, Universidad Tecnológica Equinoccial \\ Lida Katherine Sandoval Guerrero, Universidad Tecnológica Equinoccial y \\ Universidad Católica Andrés Bello (lidia.sandoval@ute.edu.ec) \\ Recibido: 21/09/2016 Aceptado: 14/12/2016
}

\begin{abstract}
Resumen
Este estudio se realizó con el fin de conocer la situación actual de los museos de la provincia del Carchi, Ecuador, que han sido citados en el Catastro de Museos y en publicaciones del Instituto Nacional de Patrimonio Cultural (INPC), mediante una evaluación integral. Para la investigación se aplicaron diversas metodologías según la variable utilizada, así el Modelo de excelencia EFQM, sustentado en la matriz de REDER para evaluar infraestructura y gestión de colecciones; en cuanto a evaluación del desempeño del personal se utilizó el Modelo por Competencias de Martha Alles. Se identificó que el museo con autonomía de gestión tiene más ventajas en el aspecto administrativo, en la creación de alianzas y captación de financiación externa, que aquellos que dependen únicamente de una institución pública: lo cual no asegura una asignación fija de recursos, por lo cual la gestión se dificulta, mostrándose falencias en infraestructura, planificación, aspecto expositivo y manejo de colecciones. La problemática se centra en la gestión del personal, pues ninguno de ellos cuenta con un sistema de contratación que exija un nivel de competencias acorde con las demandas del museo. Otros aspectos que formaron parte de la evaluación fueron las condiciones ambientales y lumínicas, en donde la temperatura se ha mantenido constante en cada museo, la humedad relativa ha sido la que muestra más fluctuaciones; en cuanto a la intensidad de la luz, en dos museos excede los límites permitidos, puede generar deterioros en los bienes si no se buscan alternativas para mejorar esta situación.
\end{abstract}

Palabras claves: gestión de museos, Competencias del Personal, Modelo de Excelencia, Administración de Colecciones.

\section{Abstract}

This study was conducted to understand the current situation of museums in the province of Carchi, Ecuador that have been mentioned in the Register of Museums and publications of the National Institute of Cultural Heritage CPI, through a global evaluation. The research used different methodologies as EFQM excellence model, based on the matrix that allowed to evaluate REDER infrastructure and collections management matrix; in terms of staffperformance Alles model was applied. It was determined that if the museum presents management autonomy, it has more advantages on the administrative side, than those that rely solely on a public institution which does not ensure a fixed resource, so management is difficult, showing gaps in infrastructure, planning, exbibition and collections management aspect. The issue focuses on personnel management, as none of them has a hiring system that requires a level of competence commensurate with the demands of the museum. Other aspects that were part of the evaluation were environmental and lighting conditions, where the temperature has remained constant in each museum, the relative bumidity, showing more fluctuations; in terms of light intensity in two museums exceeds allowable limits, potentially leading to damage of the goods if they do not seek alternatives to improve this situation.

Keywords: museum management, Staff Skills, Excellence Model, Collections Management. 


\section{INTRODUCCIÓN}

Entre tantas y variadas definiciones en torno al término "museo", el Consejo Internacional de Museos (ICOM) lo define como una institución al servicio de la comunidad, cuyos fines se centran en la conservación, exposición y transferencia del patrimonio parala educación yel deleite dela sociedad (2007, pág. 3), esta acepción es una de las más claras pues establece sus funciones y propósito. Desvallées \& Mairesse (2010) menciona que es una "institución, establecimiento olugar, generalmente concebido para proceder a la selección, el estudio y la presentación de testimonios materiales e inmateriales del individuo y su medio ambiente". (pág. 52). Al respecto, Lord y Dexter (1998), manifiestan la importancia de mantener la consecución de la misión, mandato y objetivos de este tipo de entidades, sintetizadas en el valorar y conservar el legado material e inmaterial de años de historia y mostrar a los ciudadanos el patrimonio que adquiere un significado simbólico.

Por tanto, se puede sintetizar que el rol de este tipo de entidades es de carácter educativo - formativo y social (ICOM, 2006), como se lo establece en el Código Deontológico Profesional. Corroborándose la idea del museo, según Bruninghaus (2006), como lugar idóneo para reforzar conocimientos, ofreciendo un espacio de aprendizaje, distracción y discusión, donde se establece una relación entre la Historia, la Cultura y la Sociedad por lo que la conservación y gestión de los bienes culturales es responsabilidad de los gestores culturales y de la comunidad.

Según Ladkin (2006) la Gestión de Colecciones en los museos permite que estos desarrollen sus actividades de manera óptima, por lo cual Nagel (2008) la define como "una exigencia para la identificación y control de los objetos que determina una recuperación rápida de los mismos" (pág. 8). Este proceso, tiene la ventaja de favorecer el control y manejo de las obras, pues permite identificarlas, reconocerlas a través de sus características y ubicarlas en un contexto adecuado para su apreciación. El diagnóstico es el medio para obtener información que se revelará en fichas de registros o inventarios. En este caso, Roberts (2006) recomienda realizar fichas individuales que irán acumulando información del objeto constantemente; estas son la base de investigación, y pueden ser de gran beneficio para los procesos de conservación, restauración, exposiciones y programas educativos del museo.

La Gestión de Colecciones considera el marcaje y etiquetado de los bienes culturales, como lo afirma Seguel (2008), es una tarea primordial dentro del proceso de registro, que permite relacionar a cada objeto con toda su documentación lográndose un manejo adecuado en exposiciones, rotación de obras y sobre todo se evita el robo o desaparición.

La infraestructura de las entidades museológicas se relaciona con la Gestión de Colecciones, pues estas son espacios que al no cumplir con las condiciones adecuadas favorecen el deterioro de los bienes culturales y/o patrimoniales. Los factores de incidencia en el mantenimiento de las obras son la humedad, temperatura o luminosidad relativa.

Otro de los aspectos de estudio es la Gestión del Talento Humano en los museos, tomándose como referencia Martha Alles (2006) quien se enfoca en la Dirección Estratégica de este recurso mediante tres momentos: selección, evaluación de desempeño y desarrollo, a fin de identificar si las capacidades del personal de los museos están relacionadas con los perfiles de cargos (job description). Así lo enfatiza Boylan (2006) al mencionar que el personal de un museo "representa una de las cartas de triunfo de una institución" (pág. 147), pues simplemente si no existieran las personas indicadas para la conservación, documentación y presentación de las colecciones, estas podrían deteriorarse o desaparecer irremediablemente.

Por lo cual el ICOM (2006) sostiene que en los museos debe existir un equipo multidisciplinario con formación que corresponda a la función específica que va a desempeñar, enfocándose en todos los conocimientos y experiencias que hayan adquirido. Es decir, deben poseer "competencias cardinales y específicas" (Alles, 2006) que respondan a los perfiles de cada cargo, así el ICOM (2009), señala como capacidades genéricas a la de comunicación, evaluación de métodos, análisis de datos, diseños de proyectos, tecnología de información y relaciones interpersonales. En cuanto a las competencias más específicas se mencionan las de información, dirección de colecciones y preservación, donde se aplican conocimientos acerca de la gestión de colecciones, documentación, al igual que la experiencia y conocimiento en procesos de conservación y restauración.

Si se consideran los aspectos mencionados, se puede establecer que la gestión de entidades museológicas desde el punto de vista técnico como lo establecen Alballay y Avendaño (2010), debe ajustarse a los cambios que la cultura afronta, así como a factores de influencia. Por lo cual el presente estudio se ha enfocado a analizar las condiciones de la infraestructura, las colecciones y 
la idoneidad de los capacidades del personal con los perfiles de cargos de los museos de la provincia del Carchi, registrados en Sistema Ecuatoriano de Museos y Política Nacional de Museos - SIEMEC (2011) , recogiéndose información del Catastro Nacional de Museos y resultados del diagnóstico de los museos indicados en la siguiente tabla.

Tabla 1

Museos de la provincia del Carchi según el Catastro de Museos (2011)

\begin{tabular}{|c|c|}
\hline Institución & Lugar \\
\hline $\begin{array}{c}\text { Museo Arqueológico, Arte Moderno y Contemporáneo } \\
\text { " Germán Bastidas Vaca" }\end{array}$ & Tulcán \\
\hline Museo "Carlos Emilio Grijalva"del Colegio Bolívar & Tulcán \\
\hline Museo paleontólogico del Mamut & Bolívar \\
\hline "Blas Ángel" en la ciudad de El Ángel & Espejo \\
\hline
\end{tabular}

Fuente: Catastro de Museos de la provincia del Carchi (2011)

Se ha incluido en la investigación al Museo Carlos Emilio Grijalva del Colegio Bolívar (Tulcán), debido a que ha sido considerado en la Guía de Bienes Culturales del Ecuador (INPC, 2011), y consta como una de las colecciones arqueológicas de importancia de la provincia del Carchi.

\section{METODOLOGÍA}

El presente estudio se sustenta en un proceso metodológico de carácter cualitativo (Hernández Sampieri, 2014) aplicado mediante la observación y el registro del estado actual de los museos de la provincia del Carchi. La investigación se centró en el análisis técnico de las condiciones de la infraestructura (condiciones de salas de exposición, depósito, espacios aledaños), gestión de colección (control de registro, inventario, catalogación, análisis de almacenamiento, manipulación y transporte) mediante formularios con escalas de valoración basadas en el Modelo EFQM que aplica el esquema lógico de REDER (resultados, enfoque, despliegue, evaluación y revisión) a fin de buscar la mejora continua de las entidades en estudio. La herramienta adoptada permitió evaluar cada una de los factores a partir de criterios de infraestructura como: ubicación y entorno, condiciones de espacio físico, riesgos, disposición del espacio interior; criterios de colecciones como: liderazgo, estrategia, personas, alianzas y recursos, procesos, productos y servicios, sociedad.

Finalmente, el análisis dela correlación delaformación y experiencia del personal existente en cada uno de los museos con relación a los perfiles de sus puestos de trabajo se realizó mediante la aplicación de la Metodología basada en Competencias propuesta por Martha Alles, para conocer la efectividad del evaluado en condiciones normales de trabajo y en condiciones especiales (estrés, cortos plazos de entrega).

\section{RESULTADOS}

\section{Museo Arqueológico, Arte Moderno y Contemporáneo Germán Bastidas Vaca}

Datos proporcionados por la página oficial de la Casa de laCultura Ecuatoriana "Benjamín Carrión" (2014), han permitido conocer que el Museo Arqueológico, Arte Moderno y Contemporáneo "Germán Bastidas Vaca" de la ciudad de Tulcán, es de "dependencia con autonomía de gestión" (Lord Barry y Gail Dexter, 1998) pues "depende orgánicamente de un organismo de la Administración Pública" (Billart Hernández y Tresserras, 2001, Pg. 118), es parte de la Casa de la Cultura Ecuatoriana CCE "Benjamín Carrión" Núcleo del Carchi, inaugurado el 08 de diciembre de 2006.

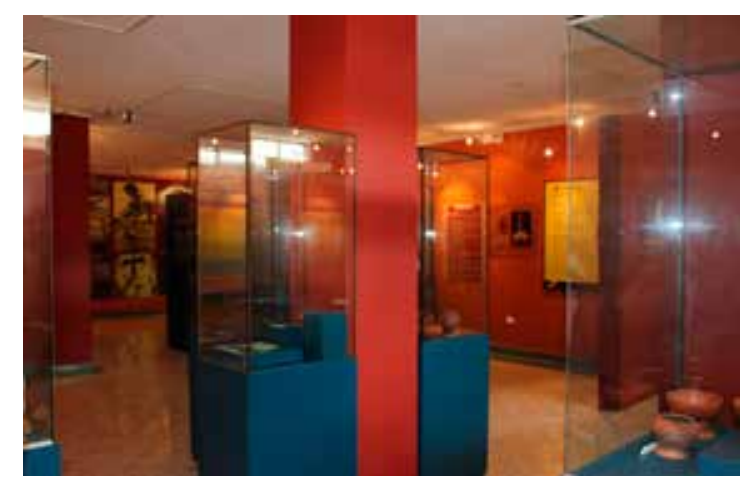

“otografía 1. Museo de Arte Moderno y Contemporáneo "Germán Bastidas Vaca"

Durante la observación realizada, se pudo constatar que el museo posee cuatro salas: en la primera sala llamada "Origen y creación", se muestran las etapas cronológicas de la independencia del Carchi y la creación de sus cantones.

En la primera parte se narra una reseña histórica de la época aborigen cuando en estos territorios habitaron los Pastos y los Quillasingas. Se narra también la situación del Carchi en la época de la República. Como parte de su población se considera el asentamiento de la comunidad Awá y afroecuatoriana; la reseña geográfica y división política también es parte de esta sala. En la segunda sala, "Fiestas y leyendas", se muestran las primeras manifestaciones culturales que responden a un pasado y presente de los pobladores de esta provincia, que son parte de su patrimonio inmaterial. 
Las leyendas ocupan un lugar de importancia en el museo, pues hacen referencia a las imágenes que se veneran en las diferentes iglesias del Carchi.

La tercera sala denominada "Arte Contemporáneo" hace referencia al proceso evolutivo y cronológico de los artistas de la provincia, efectúa un análisis desde los aspectos político, social, económico, artístico y estético que influyeron como en el período bananero, la revolución cultural y el período petrolero.

Finalmente, la sala arqueológica por su parte brinda a los visitantes un recorrido general por los períodos culturales prehispánicos del Ecuador, con énfasis en las culturas y pueblos que habitaron en lo que hoy es Carchi: Negativo del Carchi o Capulí, Tuncahuán (El Ángel o Piartal), Cuazmal (Tuza o Pasto). Resalta también la presencia inca en la provincia, describiendo las fortalezas y vestigios que se encontraron.

\section{Personal del museo}

En el nivel estratégico el museo cuenta con el cargo de Director, que lo asume el Presidente de la Casa de la Cultura - Núcleo Carchi, el mismo que coordina las acciones del museo al igual que todas las actividades culturales de esta entidad; el nivel operativo cuenta con la labor de dos personas que ocupan el cargo de guías del museo, cuya preparación académica es ajena al ámbito turístico.

Tabla 2

Personal del Museo Arqueológico, Arte Moderno y Contemporáneo "Germán Bastidas Vaca"

\begin{tabular}{|c|c|c|c|}
\hline $\begin{array}{c}\text { Nivel } \\
\text { Jerárquico }\end{array}$ & Nombre & Cargo & $\begin{array}{c}\text { Preparación } \\
\text { Académica }\end{array}$ \\
\hline $\begin{array}{c}\text { Nivel } \\
\text { Estratégico }\end{array}$ & $\begin{array}{c}\text { Jorge Ramiro } \\
\text { Almeida Almeida }\end{array}$ & $\begin{array}{l}\text { Presidente CCE } \\
\text { Núcleo Carchi }\end{array}$ & Sociólogo \\
\hline $\begin{array}{c}\text { Nivel } \\
\text { Operativo }\end{array}$ & $\begin{array}{c}\text { Cielo Albania } \\
\text { Pozo Santacruz }\end{array}$ & Guía de Museo & $\begin{array}{c}\text { Servidor público } \\
\text { de apoyo 4 }\end{array}$ \\
\cline { 2 - 4 } & $\begin{array}{c}\text { Miriam del Rocío } \\
\text { Román Lozano }\end{array}$ & Guía de Museo & $\begin{array}{c}\text { Servidor público } \\
\text { de apoyo 4 }\end{array}$ \\
\hline
\end{tabular}

Fuente: Casa de la Cultura (2013)

Autor: Elaboración propia

\section{Museo Paleontológico de Bolívar}

Tal como lo menciona Armas (2013), en el museo que está ubicado en el cantón Bolívar de la provincia del Carchi, "existen salas donde se lucen los restos fosilizados de las especies que existieron en las montañas, colinas y valles llenos de fauna que era su alimento" (pg. 33).
La Asociación de Emprendedores del cantón está a cargo del museo por un comodato de 20 años.

Dentro de su mandato se incluye la gestión de artesanías y gastronomía de Bolívar, formando un Complejo de aproximadamente $2560 \mathrm{~m}^{2}$, divididos en un Parque Temático que contiene esculturas de animales de la era cuaternaria (mastodontes, mamuts, tigres dientes de sable), una área comercial destinada a la gastronomía local, artesanías y recuerdos.

En su estructura el museo cuenta con tres salas:

Paleontológica, que alberga huesos fósiles, como colmillos, vértebras, costillas, fragmentosdecaderas

Arqueológica, donde se encuentran piezas arqueológicas, principalmente pequeñas vasijas de las fases de la Cultura Pasto: Negativo del Carchi, El Ángel y Cuasmal

Sala de proyecciones con recursos audiovisuales.

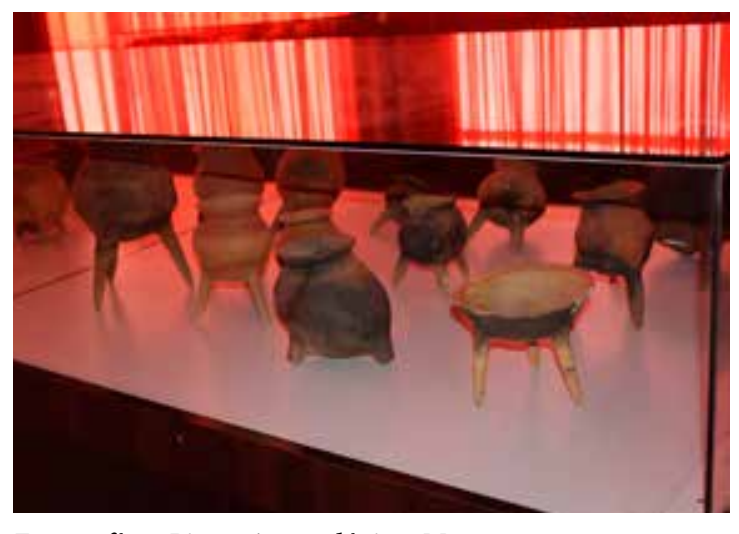

Fotografía 2. Piezas Arqueológicas Museo

Paleontológico de Bolívar

Créditos: Yajiara Chávez

\section{Personal del museo}

El Museo Paleontológico de Bolívar al ser una entidad dirigida por un organismo sin fines de lucro, cuenta con dos directivos principales, conformando el nivel estratégico de la institución, que está a cargo de la Asociación de Emprendedores de Bolívar, la administradora general y la presidenta son quienes encabezan la institución, las mismas que gestionan las acciones del museo al igual que todas las actividades que se realizan en la Asociación; ninguna de ellas posee un título universitario.

El nivel operativo cuenta con la labor de una persona que ocupa el cargo de guía del museo, y la encargada directa del mismo, cuya preparación académica tiene concordancia con el ámbito turístico. 
Tabla 3.

Personal del Museo Paleontológico de Bolívar

\begin{tabular}{|c|c|c|c|}
\hline $\begin{array}{c}\text { Nivel } \\
\text { Jerárquico }\end{array}$ & Nombre & Cargo & $\begin{array}{c}\text { Preparación } \\
\text { Académica }\end{array}$ \\
\hline $\begin{array}{c}\text { Nivel } \\
\text { Estratégico }\end{array}$ & $\begin{array}{c}\text { Jorge Ramiro } \\
\text { Almeida Almeida }\end{array}$ & $\begin{array}{c}\text { Presidente CCE } \\
\text { Núcleo Carchi }\end{array}$ & Sociólogo \\
\hline \multirow{2}{*}{$\begin{array}{c}\text { Nivel } \\
\text { Operativo }\end{array}$} & $\begin{array}{c}\text { Cielo Albania } \\
\text { Pozo Santacruz }\end{array}$ & $\begin{array}{c}\text { Presidenta de la } \\
\text { Asociación de } \\
\text { Emprendedores } \\
\text { de Bolívar }\end{array}$ & Bachiller \\
\cline { 2 - 4 } & $\begin{array}{c}\text { Miriam del Rocío } \\
\text { Román Lozano }\end{array}$ & Guía de Museo & $\begin{array}{c}\text { Egresada de } \\
\text { Aministración } \\
\text { Turística }\end{array}$ \\
\hline
\end{tabular}

Fuente: Documentos del Museo Paleontológico Autor: elaboración propia

\section{Museo “Carlos Emilio Grijalva”}

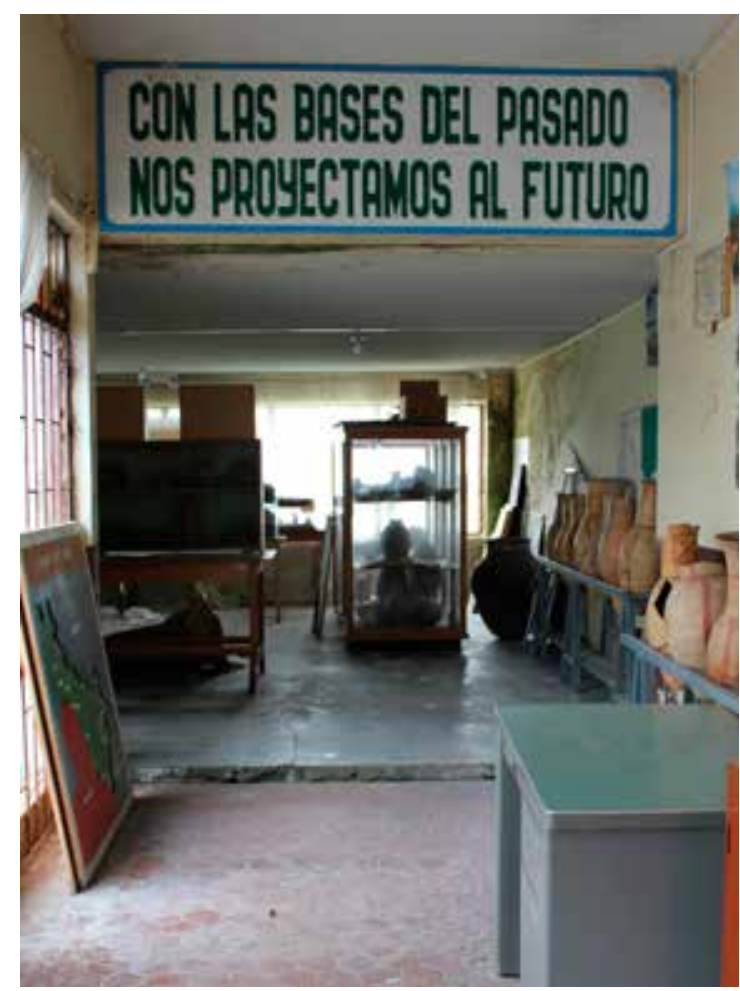

Fotografía 3. Estado actual Museo "Carlos Emilio Grijalva” Créditos: Yajiara Chávez

El Museo "Carlos Emilio Grijalva" se encuentra en la Unidad Educativa "Bolívar", en la ciudad de Tulcán, en el cual se encuentran depositadas algunas piezas arqueológicas de la cultura Pasto, y piezas paleontológicas. En lo que se refiere a la cerámica, las piezas no se encuentran organizadas, pero los investigadores de la Unidad Educativa han logrado localizar mediante estudios su ubicación de acuerdo con las diferentes fases que se encuentran en la cultura Pasto.

\section{Personal del museo}

El Museo "Carlos Emilio Grijalva" está a cargo únicamente del Rector de la Unidad Educativa "Bolívar", el mismo que posee un título de cuarto nivel superior en Educación.

\section{Museo "Blas Ángel”}

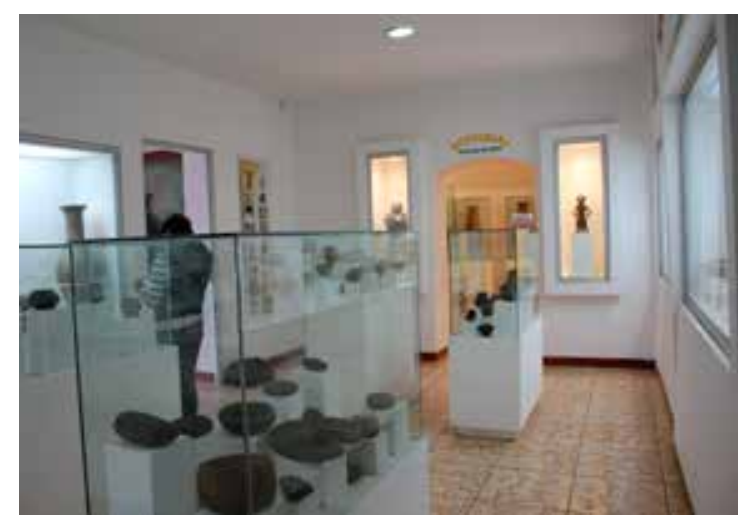

Fotografía 4. Sala Arqueológica Museo “Blas Ángel” Créditos: Yajiara Chávez

Según datos proporcionados por uno de los funcionarios del Museo "Blas Ángel", este se inauguró el3 de marzo de 1999, y pasó a funcionar en su nuevo local el 24 de abril de 2009, frente al Parque Libertad.

En este espacio se encuentran piezas valiosas de la Cultura Pasto, las cuales han sido parte de 30 años de estudios, se ha logrado ubicar las botijuelas de la fase "El Ángel", que se caracterizan por sus decoraciones antropomorfas y zoomorfas que corresponden al período de Desarrollo Regional.

El museo cuenta con una sala de Arte Moderno y Contemporáneo donde se muestran aportes de artistas como: Rafael Troya, César Villacrés y Ángel María Pozo; la sala arqueológica donde se observan las diferentes fases de evolución: Negativo del Carchi (750 - 850 d.C.), El Ángel (850 - 1250 d.C.), Cuasmal o Tuza $(1250-1534$ d.C.) y la fase de las Botijuelas de El Ángel, que son decoradas con figuras geométricas, antropomorfas y zoomorfas.

\section{Personal del museo}

El Museo "Blas Ángel" forma parte del Gobierno Autónomo Descentralizado del cantón Espejo, y está a cargo de dos miembros de la Unidad de Turismo de la Municipalidad, estos dos funcionarios son los que empezaron con las investigaciones en torno a las piezas, con el fin de instaurar el Museo "Blas Ángel", tal como se muestra en el siguiente cuadro: 
Tabla 4

Personal directivo a cargo del Museo "Carlos Emilio Grijalva

\begin{tabular}{|c|c|c|}
\hline Nombre & Cargo & Preparación Académica \\
\hline Msc.Rodrigo Sánchez & Rector de la Unidad Educativa " Bolivar" & Máster en Educación \\
\hline
\end{tabular}

Fuente: Archivos Unidad Educativa "Bolívar"

Autor: elaboración propia

Tabla 5

Personal directivo del Museo “Blas Ángel ”

\begin{tabular}{|c|c|c|}
\hline Nombre & Cargo & Preparación Académica \\
\hline Luis Antonio Ibarra & Técnico de Cultura del GAD del cantón Espejo & $\begin{array}{c}\text { Licenciado en Ciencias de } \\
\text { la Educación }\end{array}$ \\
\hline Germán Bracho Cifuentes & $\begin{array}{c}\text { Asistente de Cultura del GAD del cantón } \\
\text { Espejo persona a cargo del Museo } \\
\text { "Blas Ángel" }\end{array}$ & Tecnólogo en Turismo \\
\hline
\end{tabular}

Fuente: Archivos GAD Municipal de Espejo

Autor: elaboración propia

Infraestructura y gestión de colecciones

Los resultados obtenidos de la matriz de REDER permitieron evidenciar la realidad actual de los museos en referencia a las variables de infraestructura y gestión de colecciones bajo diversos criterios, como se aprecia en la Tabla 6.

A continuación se analizan las dimensiones y criterios señalados:

\section{Infraestructura}

\section{Criterio 1: Ubicación y entorno}

Tras respectivas valoraciones, el Museo "Blas Ánge" y el Arqueológico, Arte Moderno y Contemporáneo "Germán Bastidas Vaca", superan el 95\%, el primero es el de mayor puntaje (97,92\%), mientras que los museos "Carlos Emilio Grijalva" y Paleontológico de Bolívar, han obtenido menos del 90\%.

\section{Criterio 2: Condiciones del espacio físico}

Tras la evaluación, se muestra que los museos Paleontológico de Bolívar, Arqueológico, Arte ModernoyContemporáneo "Germán Bastidas Vaca" y "BlasÁngel"superanel90\%; peroporsuparte, el Museo "Carlos Emilio Grijalva", ha obtenido menos de 50\%.

\section{Criterio 3: Riesgos}

Se han obtenido datos que demuestran que el museo conmayor porcentajeeselArqueológico, ArteModerno y Contemporáneo "Germán Bastidas Vaca" (70\%), y que además, el museo que posee menos puntuación es el Museo "Carlos Emilio Grijalva" (17,38\%). Los demás se encuentran en un rango de $60-70 \%$.

\section{Criterio 4: Disposición del espacio interior}

Al evaluar a cada museo en cuanto a la disposición de las colecciones, se ha podido determinar que el Museo Paleontológico de Bolívar ha obtenido $100 \%$ en este criterio, mientras que el Museo "Carlos Emilio Grijalva" ha alcanzado resultados inferiores a lo esperado, de 8,33\% en este aspecto. Los demás se encuentran en un rango de 75-90\%.

\section{Gestión de Colecciones}

\section{Criterio 1: Liderazgo}

Se ha determinado que los museos "Blas Ángel", Arqueológico, Arte Moderno y Contemporáneo "Germán Bastidas Vaca" y Paleontológico de Bolívar han obtenido un porcentajeen un rango de $30-35 \%$; y el Museo "Carlos Emilio Grijalva" ha obtenido 12,08\%.

\section{Criterio 2: Estrategia}

Tras la respectiva evaluación se demuestra que los museos "Blas Ángel”, Arqueológico, Arte Moderno 
Tabla 6

Tabla comparativa entre museos

\begin{tabular}{|c|c|c|c|c|c|c|}
\hline & & $\begin{array}{r} \\
\text { Arte N } \\
\text { "C }\end{array}$ & 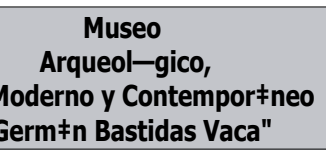 & $\begin{array}{c}\text { Museo } \\
\begin{array}{c}\text { Paleontol-gico de } \\
\text { Bol'var }\end{array}\end{array}$ & $\begin{array}{c}\text { Museo } \\
\text { ÒCarlos Emilio } \\
\text { Grijalvaó }\end{array}$ & $\begin{array}{c}\text { Museo } \\
\text { ÒEmilio çngeló }\end{array}$ \\
\hline \multicolumn{7}{|c|}{ INFRAESTRUCTURA } \\
\hline \multicolumn{2}{|l|}{ Criterio 1: Unicaci-n y entorno } & & $1(96,17 \%)$ & $2(88,08 \%)$ & $2(89,83 \%)$ & $1(97.92 \%)$ \\
\hline \multicolumn{2}{|l|}{$\begin{array}{l}\text { Criterio 2: Condiciones del } \\
\text { espacio f'sico }\end{array}$} & & $1(94,49 \%)$ & $1(96,88 \%)$ & $3(47,81 \%)$ & $1(92.14 \%)$ \\
\hline \multicolumn{2}{|l|}{ Criterio 3: Riesgos } & & $2(70,00 \%)$ & $3(61,31 \%)$ & $4(17,38 \%)$ & $3(63.81 \%)$ \\
\hline \multicolumn{2}{|l|}{$\begin{array}{l}\text { Criterio 4: Disposici-n del } \\
\text { espacio f's'co }\end{array}$} & & $2(87,50 \%)$ & $1(100 \%)$ & $5(8,33 \%)$ & $2(75.00 \%)$ \\
\hline \multicolumn{7}{|c|}{ GESTIÎN COLECCIONES } \\
\hline \multicolumn{2}{|l|}{ Criterio 1: Liderazgo } & & $4(35,00 \%)$ & $4(35,42 \%)$ & $5(12,08 \%)$ & $4(35,42 \%)$ \\
\hline \multicolumn{2}{|l|}{ Criterio 2: EstratŽgia } & & $3(57,92 \%)$ & $3(50,04 \%)$ & $5(14,75 \%)$ & $3(50,04 \%)$ \\
\hline \multicolumn{2}{|l|}{ Criterio 3: Personas } & & $3(59,79 \%)$ & $3(49,31 \%)$ & $4(18,92 \%)$ & $3(49,31 \%)$ \\
\hline \multicolumn{2}{|l|}{ Criterio 4: Riesgos } & & $3(46,49 \%)$ & $4(15,59 \%)$ & $5(3,82 \%)$ & $4(15,59 \%)$ \\
\hline \multicolumn{2}{|l|}{$\begin{array}{l}\text { Criterio 5: Procesos, } \\
\text { productos y servicios }\end{array}$} & & $4(21,88 \%)$ & $5(9,48 \%)$ & $5(0,63 \%)$ & $5(9,48 \%)$ \\
\hline \multicolumn{2}{|l|}{$\begin{array}{l}\text { Criterio 6: Resultados en la } \\
\text { sociedad }\end{array}$} & & $2(71,43 \%)$ & $3(63,10 \%)$ & $4(32,14 \%)$ & $3(63,10 \%)$ \\
\hline \multicolumn{7}{|c|}{$90-100 \%$} \\
\hline 2 & \multicolumn{2}{|c|}{$65-89 \%$} & \multicolumn{4}{|c|}{ Destacado,cumple con la mayor parte de requerimientos esenciales } \\
\hline 3 & \multicolumn{2}{|c|}{$40-64 \%$} & \multicolumn{4}{|c|}{ Regula, se puede demostrar cumplimiento parcial de requerimientos } \\
\hline 4 & \multicolumn{2}{|c|}{$15-39 \%$} & \multicolumn{4}{|c|}{ Necesita mejorar, limitada capacidad para cumplir con requerimientos } \\
\hline 5 & \multicolumn{2}{|c|}{$0-14$} & \multicolumn{4}{|c|}{ Resultados inferiores a lo esperado } \\
\hline
\end{tabular}

Fuente: Archivos Unidad Educativa "Bolívar"

Autor: elaboración propia

y Contemporáneo "Germán Bastidas Vaca" y Paleontológico de Bolívar han obtenido porcentajes entre 40-60\%; mientras que el Museo "Carlos Emilio Grijalva" ha obtenido un porcentaje de $14,75 \%$.

\section{Criterio 3: Personas}

Tras evaluar, se ha determinado que los museos "Blas Ángel", Arqueológico, Arte Moderno y Contemporáneo "Germán Bastidas Vaca" y Paleontológico de Bolívar han obtenido porcentajes entre 40 - 60\%; mientras que el Museo "Carlos Emilio Grijalva" obtuvo un porcentaje menor a 20\%.

\section{Criterio 4: Alianzas y recursos}

Seha obtenidocomo resultados queen primerlugar, el MuseoArqueológico,ArteModernoyContemporáneo "Germán Bastidas Vaca" ha obtenido 46,49\%, mientras que el Museo "Carlos Emilio Grijalva" obtuvo 3,82\%. Los museos "Blas Ángel" y Paleontológico de Bolívar se encuentran en un rango de 15-20\%.

\section{Criterio 5: Procesos, productos y servicios}

Al evaluar, se ha determinado que el Museo Arqueológico, Arte Moderno y Contemporáneo "Germán Bastidas Vaca" tiene un porcentaje de 21,88\%, mientras que los demás museos han obtenido resultados inferiores, en un rango de 0 - 10\%, el Museo "Carlos Emilio Grijalva" es el de menor puntuación (0,63\%).

\section{Criterio 6: Resultados en la sociedad}

El Museo Arqueológico, Arte Moderno y Contemporáneo "Germán Bastidas Vaca" 
obtuvo 71,43\%, mientras que el Museo "Carlos Emilio Grijalva", 32,14\%. Los museos "Blas Ángel” y Paleontológico de Bolívar cumplen con porcentajes en un rango de $60-70 \%$.

\section{DISCUSIÓN}

De manera general se pudo llegar a determinar que la situación de los Museos de la provincia del Carchi, no son del todo deseables pues presentan muchas falencias en cuanto a las variables analizadas; se puede generalizar que la entidad museológica que tiene autonomía de gestión se encuentra en mejores condiciones, deja en evidencia la carencia de recursos en los otros museos y la falta de inversión en mejorar su estado actual.

Es así que en infraestructura, tras la evaluación de las características de los elementos constructivos del edificio y la preparación que tienen los museos frente a los posibles riesgos estructurales, las valoraciones muestran que los museos Paleontológico de Bolívar, Arqueológico, Arte Moderno y Contemporáneo "Germán Bastidas Vaca" y "Blas Ángel", han cumplido excepcionalmente con los requerimientos en este criterio; pero por su parte, el Museo "Carlos Emilio Grijalva", ha obtenido menos de 50\%, demostrando que el cumplimiento de los requerimientos es parcial, pues tanto las paredes como los pisos poseen muestras de filtraciones y fisuras.

En la evaluación de la ubicación del edificio en la cual funcionan los museos, y el entorno en el que se encuentran, el Museo "Blas Ángel" y el Arqueológico, Arte Moderno y Contemporáneo "Germán Bastidas Vaca", han cumplido excepcionalmente con las exigencias en este aspecto, pues están alejados de cualquier sitio de riesgo, mientras que los museos "Carlos Emilio Grijalva" y Paleontológico de Bolívar, han disminuido su puntaje debido a que el primero está cercano a un terreno baldío donde se muestra vegetación que provoca residuos en el interior; y el segundo está ubicado en la Panamericana Norte, lo cual genera un alto nivel de contaminación atmosférica y sonora.

Al evaluar el grado de desarrollo de la gestión de las colecciones, se ha determinado que el museo Arqueológico, Arte Moderno y Contemporáneo "Germán Bastidas Vaca", al formar parte de la CCE Benjamín Carrión que es una institución con autonomía de gestión, goza de mayor libertad para buscar alianzas y financiación pública o privada, utiliza sus ingresos para su beneficio y sostiene una normativa clara que debe cumplirse. Los museos "Blas Ángel", y Paleontológico de Bolívar tienen una limitada capacidad para cumplir con los requerimientos esenciales de este aspecto, pues dependen directamente de los municipios a los cuales pertenecen, los mismos que no asignan un presupuesto fijo; como resultado de ello, estos museos se ven poco incentivados para proporcionar al público un mejor servicio. Por su parte, el Museo "Carlos Emilio Grijalva" ha obtenido resultados inferiores a lo que se solicita, pues en la actualidad la institución educativa a la que pertenece no ha establecido un área que se haga cargo del museo, y por ende ha cerrado sus puertas, dejándolo ala deriva.

En la evaluación por competencias al personal de cada museo se ha definido que ninguno de ellos cuenta con personal especializado en temas referentes a museos o conservación de bienes. Existen casos como el museo Paleontológico de Bolívar y el "Blas Ángel", en los cuales las personas que están directamente a cargo tienen relación con el aspecto turístico, pero no conocen sobre el manejo de las colecciones. Por su parte, el museo Arqueológico, de Arte Moderno y Contemporáneo "Germán Bastidas Vaca" cuenta con un personal operativo que posee un título incompatible con lo que se requiere para manejar un museo. Tomando en cuenta el personal directivo, únicamente el Museo Arqueológico, de Arte Moderno yContemporáneo "Germán Bastidas Vaca" y el museo "Blas Ángel" tienen a la cabeza un funcionario que conoce sobre el movimiento cultural en el cual está inmerso el museo; mientras que los demás no cumplen con las competencias que se requieren para estar al mando de una institución museística; pues en el museo Paleontológico, los directivos no poseen título universitario; en el museo "Carlos Emilio Grijalva", nadie tiene una responsabilidad directa, porque cuando cerró sus puertas, quedó en manos del rector de la institución educativa, pero ha quedado en el abandono al no planificarse ningún tipo de proyecto referente al mismo.

Las condiciones ambientales y lumínicas, en el museo "Blas Ángel", mantienen un promedio de temperatura ligeramente más bajo del rango óptimo, pero no representan ningún riesgo al mantenerse oscilaciones únicamente de $+/-2$. En la sala de Arte Moderno y Contemporáneo, en cuanto a humedad relativa, el promedio es de 59,117\%, con fluctuaciones que superan los $+/-10$, en este caso, existen momentos del día en el cual se excede el porcentaje en comparación al rango óptimo que se debe mantener, pero las oscilaciones son las que podrían generar craqueladuras en las pinturas, las cuales ya se empiezan a mostrar. En la intensidad de la luz, se ha observado que se incrementa al mediodía, pero no excededel rangoóptimoy porendeno produce daños. 
En la sala de Arqueología, a pesar que la temperatura es ligeramente más baja del rango permitido con objetos de cerámica, no ha generado daños; la humedad relativa si se ha excedido, pues el límite es $60 \%$, y en este caso, el porcentaje promedio es de $64,858 \%$, llegando incluso a superar el $68 \%$, pero aun así, no se muestran deterioros biológicos o mecánicos en los objetos expuestos. La luz no representa un problema, pues al ser luz artificial, puede ser controlada con facilidad.

En el Museo Paleontológico de Bolívar, se encuentran dos escenarios, pues en la Sala de Arqueología se mantienen las condiciones ambientales y lumínicas adecuadas, respetando los rangos óptimos; y en la sala de Paleontología, el problema radica en la intensidad de la luz, pues en horas de la mañana se registra un gran incremento, llegando a superar los 1000 luxes, pues una de las vitrinas de esta sala en la cual se colocó el sensor está cercana a una ventana que no está cubierta totalmente; los huesos pueden recibir máximo 50 luxes, y en este caso se supera abismalmente, incluso el promedio diario es de 154 luxes; posiblemente es uno de los factores que provocanlasmanchasenelmaterialóseoquese exhibe.

En el Museo "Carlos Emilio Grijalva", las condiciones lumínicas son las más preocupantes, pues en los sitios cercanos a las ventanas donde se colocaron los sensores se ha registrado que en horas de la mañana supera los 20000 luxes, lo cual es extremo y perjudicial para los objetos cerámicos, que pueden recibir hasta 300 luxes, lo cual es posiblemente un factor del desecamiento y debilitamiento estructural de muchas de las piezas exhibidas. Además, las oscilaciones en la humedad relativa, provocan algunos deterioros físicos y biológicos en el edificio.

En el Museo Arqueológico, Arte Moderno y Contemporáneo "Germán Bastidas Vaca", la temperatura es constante, no se muestran oscilaciones. En humedad relativa, el promedio encaja dentro del rango óptimo, pero tanto en la sala de Arqueología, en la de Arte Moderno/ Contemporáneo y en la sala temporal, se presencian fluctuaciones de aproximadamente $+/-10 \%$, pero con estos porcentajes no se produce ningún daño severo. Tomando en cuenta la intensidad de la luz, en la sala de Arqueología, donde existe una pequeña ventana se registra un promedio de 99,2 luxes, que está en un rango óptimo, aunque al mediodía se registra un incremento considerable. En la sala de Arte Moderno y Contemporáneo, donde se guardan pinturas de caballete, la intensidad de la luz se mantiene en el rango adecuado, al igual que en la sala de exposiciones temporales.

\section{REFERENCIAS BIBLIOGRÁFICAS}

Alballay, S., \& Avendaño, C. (2010). Gestión Cultural: Entre conceptos lejanos y realidades cercanas. Argentina: EDUVIM.

Alles, M. (2006). Desarrollo del talento bumano basado en competencias. (2da). Buenos Aires, Argentina: Granica.

Armas, M. (2013). Desarrollo de una campaña de bien público para difundir información sobre el Museo Paleontológico de Bolívar. Ibarra, Imbabura, Ecuador.

Ballart, Hernández Josep y Tresserras, Jordi Juan I (2011) Gestión del patrimonio, (1º edición ed.)., Barcelona.

Boylan, P. (2006). Gestión de los efectivos. En UNESCO/ICOM, Cómo administrar un museo: Manual práctico (págs. 147 - 160). París: UNESCO/ ICOM.

Bruninghaus, C. (2006). La misión educativa del museo en el marco de las funciones museísticas. En UNESCO, Cómo administrar un museo: Manual práctico (págs. 127 - 140). París: UNESCO - ICOM.

Carchi, P. (2015).Prefectura del Gobierno Provincial del Carchi. Recuperado el 17 de julio de 2015, de Información Provincial: http://carchi.gob.ec/index. php/nuestra-provincia/informacion-provincial

CCE. (2013). Casa de la Cultura Ecuatoriana. Recuperado el 01 de 07 de 2015, de http://www. casadelacultura.gob.ec/archivosleytransparencia/ directorio_instituciona_enero_2014(2).pdf

CCE. (2014). Casa de la Cultura Ecuatoriana "Benjamín Carrión". Recuperado el 18 de julio de 2015, de Museos CCE: http://www. casadelacultura.gob.ec/?ar id $=3 \& \mathrm{mu}$ id $=4$ \&palabrasclave $=$ Museo Arqueológico, de Arte Moderno y Contemporáneo\&title $=$ Museo Arqueológico, de Arte Moderno y Contemporáneo

CCE. (2014). Casa dela Cultura Ecuatoriana "Benjamín Carrión". Recuperado el 1 de Julio de 2015, de Archivos CCE: http://www.casadelacultura.gob.ec/ archivosleytransparencia/directorio_instituciona_ enero_2014(2).pdf

Desvallées, A., \& Mairesse, F. (2010). Conceptos claves de museología. París: Armand Colin ICOM. 
Hernández Sampieri, C. y. (2014). Metodología de la Investigación ( $6^{\circ}$ edición ed.). México D.F.: Mc Graw Hill Interamericana.

ICOM. (2006). Código de Deontologia Profesional del ICOM. Argentina.

INPC. (2011). Guía de Bienes Culturales delEcuador (Carchi). Quito, Pichincha, Ecuador.

Ladkin, N. (2006). Gestión de colecciones. En UNESCO, Cómo administrar un museo: Manual práctico (págs. 17 - 30). París: UNESCO - ICOM.

Lord Barry y Gail Dexter. (1998). Manual de Gestión de Museos. Madrid: Ariel.

Nagel, L. (2008). Registro y Documentación. En I. Getty, Manual de Registro y Documentación de Bienes Culturales (págs. 8 -9). Santiago de Chile: Dibam.
Roberts, A. (2006). Inventarios y documentación. En UNESCO/ICOM, Cómo administrar un museo: Manual práctico (págs. 31 - 50). París: UNESCO/ ICOM.

Seguel, R. (2008). Marcaje de Bienes Culturales. En I. Getty, Manual de Registro y Documentación de Bienes Culturales (págs. 22 -29). Santiago de Chile: DIBAM

SIEMEC. (2011).Propuesta del Sistema Ecuatoriano deMuseosy Política NacionaldeMuseos. Recuperado el 08 de 12 de 2014, de http:

Turismo, M. d. (02 de diciembre de 2013). Ministerio de Turismo. Recuperado el 19 de julio de 2015, de Noticias: http://www.turismo.gob.ec/el-complejopaleontologico-mas-importante-del-norteecuatoriano-se-construye-en-carchi/ 